\title{
Cloning, Tissue Expression, and Mapping of a Human Photolyase Homolog with Similarity to Plant Blue-Light Receptors
}

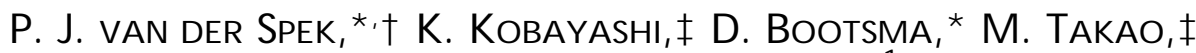 \\ A. P. M. EKER, ${ }^{*}$ AND A. YASUI ${ }^{1}$ \\ * Department of Cell Biology and Genetics, M GC, Erasmus University Rotterdam, 3000 DR Rotterdam, The Netherlands; \\ †Department of Radiotherapy, Division of Clinical Radiobiology, Dr. Daniel den Hoed Center, 3075 EA Rotterdam, \\ The Netherlands; and ¥Institute of Development, Aging and Cancer, Tohoku University, Sendai 980-77, Japan
}

Received May 13, 1996; accepted August 7, 1996

Enzymatic photoreactivation is a DNA repair mechanism that removes UV-induced pyrimidine dimer lesions by action of a single enzyme, photolyase, and visible light. Its presence has been demonstrated in a wide variety of organisms, ranging from simple prokaryotes to higher eukaryotes. We have isolated a human gene encoding a 66-kDa protein that shows clear overall homology to known bacterial photolyase genes. The human gene product is more similar to plant blue-light receptors within class I photolyases than to higher eukaryote class II photolyases. Northern blot analysis showed two transcripts with constitutive expression in all tissues examined and an elevated expression in testis. In situ hybridization with a CDNA-derived probe localized this human gene to chromosome 12q23q24.1. Southern analysis of the cloned human gene suggests a wide distribution of the gene family in various species. 1996 Academic Press, Inc.

\section{INTRODUCTION}

Photoreactivation (PHR) is a repair mechanism that removes UV-induced DNA damage by action of a single enzyme: photolyase. These lesions can interfere with processes such as replication and transcription and therefore affect viability and functional integrity of living cells. Illumination with visible light of photolyase bound to a pyrimidine dimer lesion leads to conversion of this lesion into two pyrimidine monomers. Photoreactivation of cyclobutanetype pyrimidine dimers (CPD) has been known of for a long time, and at least 15 photolyase genes have been cloned. More recently, the presence of a photolyase specific for (6-4)photoproducts in insects and plants was reported (Todo et al., 1993; Chen et al., 1994).

Sequence data from this article have been deposited with the DDJ B Database under Accession No. D84657.

${ }^{1}$ To whom correspondence should be addressed at Institute of Development, Aging and Cancer, Tohoku University, 4-1 Seiryomachi Aoba-Ku, Sendai 980-77, J apan. Telephone: 22-717-8562. Fax: 22717-8567.
Most CPD photolyases contain two chromophores, one of which is reduced FAD and essential for repair activity. The second chromophore, either 5,10-methenyltetrahydrofolate (MTHF) or 8-hydroxy-5-deazaflavin (8-HDF), works as an efficient antenna cofactor to gather light as energy supply for the dimer splitting reaction sensitized by the reduced FAD cofactor (Sancar, 1994; Eker et al., 1988). Thus far, two distantly related classes of CPD photolyases, which can be distinguished by their amino acid sequence, have been identified. Class I contains most microbial photolyases as well as several plant blue-light photoreceptors, while in dass II higher eukaryote photolyases are found (Yasui et al., 1994).

Photolyases are present in cells from many species, ranging from bacteria and yeasts to aplacental mammals like the opossum (Monodel phis domesticum) and rat kangaroo (Potorous tridactylis) (Kato et al., 1994; Yasui et al., 1994). In contrast, contradictory results have been obtained for placental mammals (see, e.g., Li et al., 1993; Sutherland and Bennett, 1995), and a corresponding photolyase gene has not been identified. Here we report the cloning, expression analysis, and chromosomal localization of a human gene that has clear homology to class I microbial photolyases.

\section{MATERIALS AND METHODS}

Identification of R19031 and cloning of cDNA. The amino acid sequence of Anacystis nidulans photolyase was used to perform a sequence comparison in the expressed sequence tag (EST) database. The NCBI TBLASTN program (Altschul et al., 1990) identified a CDNA clone (R19031, I mage Consortium) with an open reading frame homologous to the photolyase sequence used as a query. The cloned sequence was extended by PCR using a human testis library (BRL).

Sequencing and analysis of DNA and encoded proteins. DNA sequencing was carried out by the dideoxynucleotidechain-terminating procedure with fluorescent-tag-labeled deoxynucleotides using a SHIMAZU DSQ-1000. Sequences were compared for homology to other sequences in the GenBank, GenEMBL, and dBEST databases by using the BLAST software package from the NCBI server.

Chromosomal localization. In situ hybridization was performed with a biotin-labeled probe derived from the R19031 partial CDNA clone (Pinkel et al., 1986). After incubation with avidin D-FITC (Vector, USA), the probe was visualized by FISH. A representative 
S. C. FEOYHSTNKKYYPRPITRTGAN . OFNNK. SRAKPMEIVEKL . . . OKKOKTSFFN . . . VSTAMHWFR

E. C.

A. $\mathbf{n}$.

C. $r$.

H. S .

P.t.

S.c.

Nis

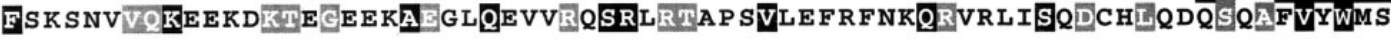

E.C.

A.n.

C. $r$.

H.s.

P.t.

s.c.

E.c.

A.n.

C. $r$.

H. $\mathbf{s}$.

P.t.

s.c.

E.c.

A. $n$.

C. $r$.

H.s.
P.t.

s.c.

E.c.

A.n.

C. $r$.

H. $\mathbf{s}$.

P. $t$.

S.c.

E.C.

A.n.

c. $r$.

H. $\mathbf{s}$.

P.t.

S.c.

E.C.

A.n.

C.r.

H. S .

P.t.

S.C.

E.C.

A.n.

C.r.

H. S .

P. t.

S. C.

E. C.

A.n.

C.r. APWKA PASVLAAADV̈ELCENY PLPI ITRSDAKANVDYACGVLEKSAVAPTGSESSGRYPYRAPTYPNAGG

H.S. DPWNAPEGIQKVAKCLIGVNYPKPMVNHAEA.SRLNI . ERMKQIYQQLSR. YRGLGLLA. SVPSNPN. . G

P. . . . . . . . . . MNYAGCKRKFDVA. EFERK.YSPAD*

S. . .

E.C.

A.n.

C.r. GGGASGGVDGAGSSGGNPTAAMGASGGAGPSSGTGTGGQGGAFRGRGDGGGSAPVSQQGGMLPPGVAVCV

H.S. NG.GF . .M. GY.SAENIP....G.CSSSG.SCSQGSGIL..HY.AHGD...... SQQTHLLRQGRS.SM

P.t.

S. C.

E.c.

A.n.

C. $r$. TAGTGGTQPDSRTVSGAHAGVSNSAGGVACDMPPPS - >

H.S . GTGLSGGKRPSQE. EDTQ. SIG. PK . . V. . QRQSTN *

P. $t$. 


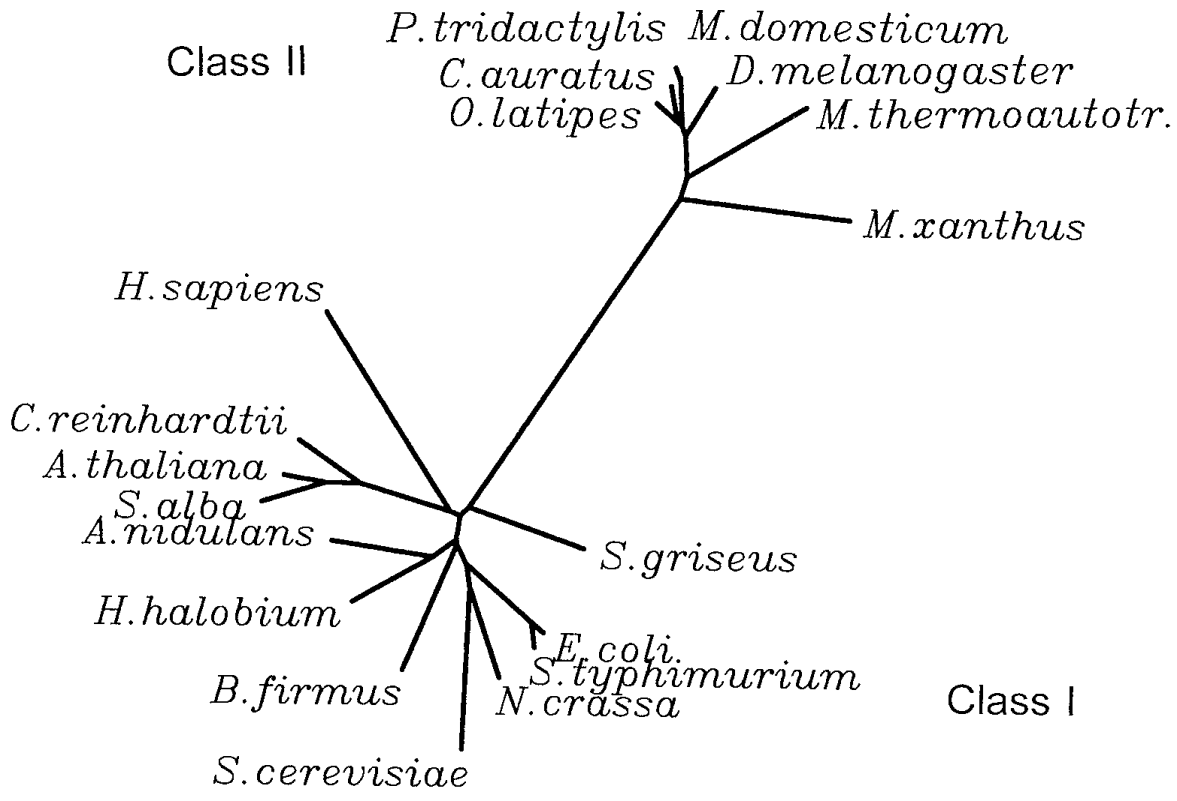

FIG. 2. Unrooted phylogenetic tree for photolyases and photolyase-like blue-light receptors from Bacillus firmus (bacterium), Escherichia coli (bacterium), Saccharomyces cerevisiae (yeast), and Salmonella typhimurium (bacterium) (class I MTHF-type photolyases); Anacystis nidulans (cyanobacterium), Halobacterium halobium (archaeon), and Streptomyces griseus (bacterium) (class I 8-HDF-type photolyases); Arabidopsis thaliana (plant), Chlamidomonas reinhardtii (green alga), and Sinapis alba (plant) (class I photolyase-like blue-light receptors); Carassius auratus (fish), Drosophila melanogaster (insect), Methanobacterium thermoautotrophicum (archaeon), Monodel phis domesticum (marsupial), Myxococcus xanthus (bacterium), Oryzias latipes (fish), and Potorous tridactylis (marsupial) (Class II photolyase).

set of more than 50 metaphases was analyzed to determine the site of hybridization.

Northern bl ot analysis. A tissue Northern blot containing $2 \mu \mathrm{g}$ of $\operatorname{poly}(\mathrm{A})^{+}$RNA per lane (Clontech human multiple-tissue Northern blot MTN I and II) was hybridized to ${ }^{32} \mathrm{P}$-labeled DNA probes using hybridization buffer (Clontech Express $\mathrm{Hyb}$ ) under conditions recommended by the supplier. A 1.2-kb cDNA probe of the cloned human gene and human $\beta$-actin cDNA were used as probes.

Southern blot analysis. Genomic DNA (10 $\mu \mathrm{g})$ from various sources was digested with EcoRI and hybridized with a 1.2-kb cDNA probe of the cloned human photolyaselike gene using Quick Hyb hybridization solution (Stratagene). Washing was done with $2 \times$ SSC $(0.1 \% \mathrm{SDS})$ at room temperature for $30 \mathrm{~min}$ and subsequently with $0.1 \times \mathrm{SSC}(0.1 \% \mathrm{SDS})$ at $68^{\circ} \mathrm{C}$ (stringent wash) or $60^{\circ} \mathrm{C}$ (less stringent wash) for $30 \mathrm{~min}$.

Phylogenetic tree An unrooted phylogenetic tree was obtained by the neighbor-joining method (PHYLIP software package, Felsenstein, 1993) using pairwise distances calculated with the Dayhoff PAM 001 matrix from a stretch of 468 amino acid positions present in all photolyases after alignment with ClustalW. Amino acid sequences of photolyases were taken from Ahmad and Cashmore (1993) (Arabidopsis thaliana); Malhotra et al. (1994) (Bacillus firmus); Kato \& al. (1994) (M. domesticum); Small et al. (1995) (Chlamydomonas reinhardtii), O'Connor \& al. (1996) (Myxococcus xanthus), and Yasui et al. (1994) (other species).

\section{RESULTS AND DISCUSSION}

A human photolyase homolog was identified by performing a TBLASTN search of the NCBI expressed se- quence tag (CDNA) database using the amino acid sequence of $A$. nidulans photolyase as query. An unpublished EST clone R19031, derived from the Soares infant brain library (Soares et al., 1994), appeared to be homologous to the query sequence. This partial CDNA was cloned from a gridded CDNA library and sequenced. 5' and 3' extensions of R19031 were obtained by nested polymerase chain reaction using a human testis library as substrateDNA source. The presence of full-length CDNA was confirmed by amplification using oligonudeotide primers covering the putative start and stop codons.

The doned DNA fragment contained an open reading frame encoding a protein of 586 amino acids. Figure 1 shows a comparison of the deduced amino acid sequences with those of various photolyases. The human gene has substantial homology (around $25 \%$ identical) to class I photolyases. Interestingly, the homology to higher eukaryote photolyases in dass II is less pronounced (around $15 \%$ identical). Furthermore, several tryptophan residues conserved among photolyases are also present in the human homolog. Among them are W277 of Escherichia coli photolyase, which plays a role in substrate binding as well as direct dimer splitting (Kim \& al., 1992), and W306 of $\mathrm{E}$. coli photolyase, which is necessary for photoreduction of the FAD chromophore (Li \& al., 1991). The conser-

FIG. 1. Alignment of amino acid sequences of photolyases from the following species: Saccharomyces cerevisiae (S.c.) (class I photolyase, MTHF type); Escherichia coli (E.c.) (class I photolyase, MTHF type); Anacystis nidulans (A.n.) (class I photolyase, 8-HDF type); Chlamydomonas reinhardtii (C.r.) (class I blue-light receptor); Homo sapiens (H.s.); and Potorous tridactylis (P.t.) (class II photolyase). Identical amino acids are shown as black boxes, whereas similar residues (A, S, T, P; D, E, N, Q; R, K; I, L, M, V; F, Y, W) are indicated by gray boxes. Tryptophan residues 277 and 306 are indicated with stars. 

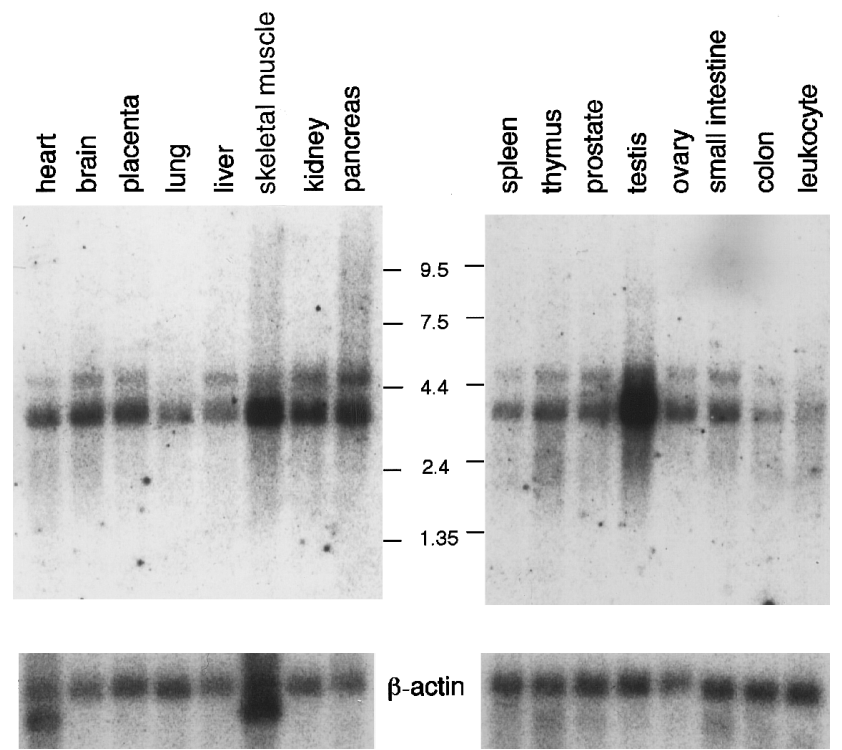

$\beta$-actin

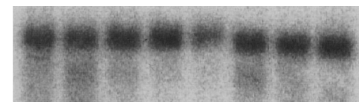

FIG. 3. Northern blot analysis of different human tissues for mRNA expression of the human photolyase homolog.

vation of tryptophan residues suggests that the human homolog has FAD binding ability.

\section{Homology to BlueLight Receptors}

Not only the overall similarity but also several features of the deduced amino acid sequence suggest that the cloned human gene might be related to photolyaselike blue-light receptors. Both class I and class II eu-

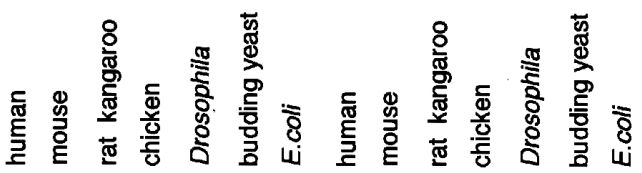

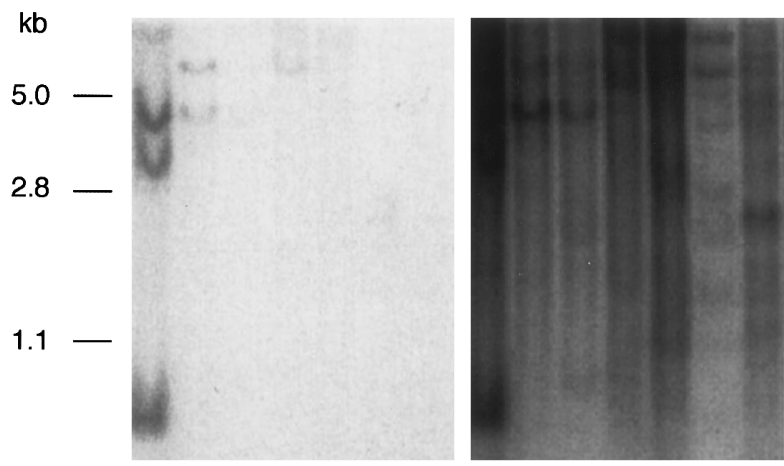

High stringent wash $\left(68^{\circ} \mathrm{C}\right) \quad$ Low stringent wash $\left(60^{\circ} \mathrm{C}\right)$

FIG. 5. Southern analysis of genomic DNA from various organisms using human CDNA as probe.

karyote photolyases possess an amino-terminal extension, which was shown to be necessary for protein transport into nucleus and mitochondria (Yasui et al., 1992). The putative human protein, however, does not have this extension, as is the case for the plant bluelight receptor family. Instead, a carboxyl-terminal extension is present, which is also found in the Arabidopsis bluelight receptor gene HY4 (Ahmad and Cashmore, 1993) and in the putative blue-light receptor of $\mathrm{C}$. reinhardti $\mathrm{i}$
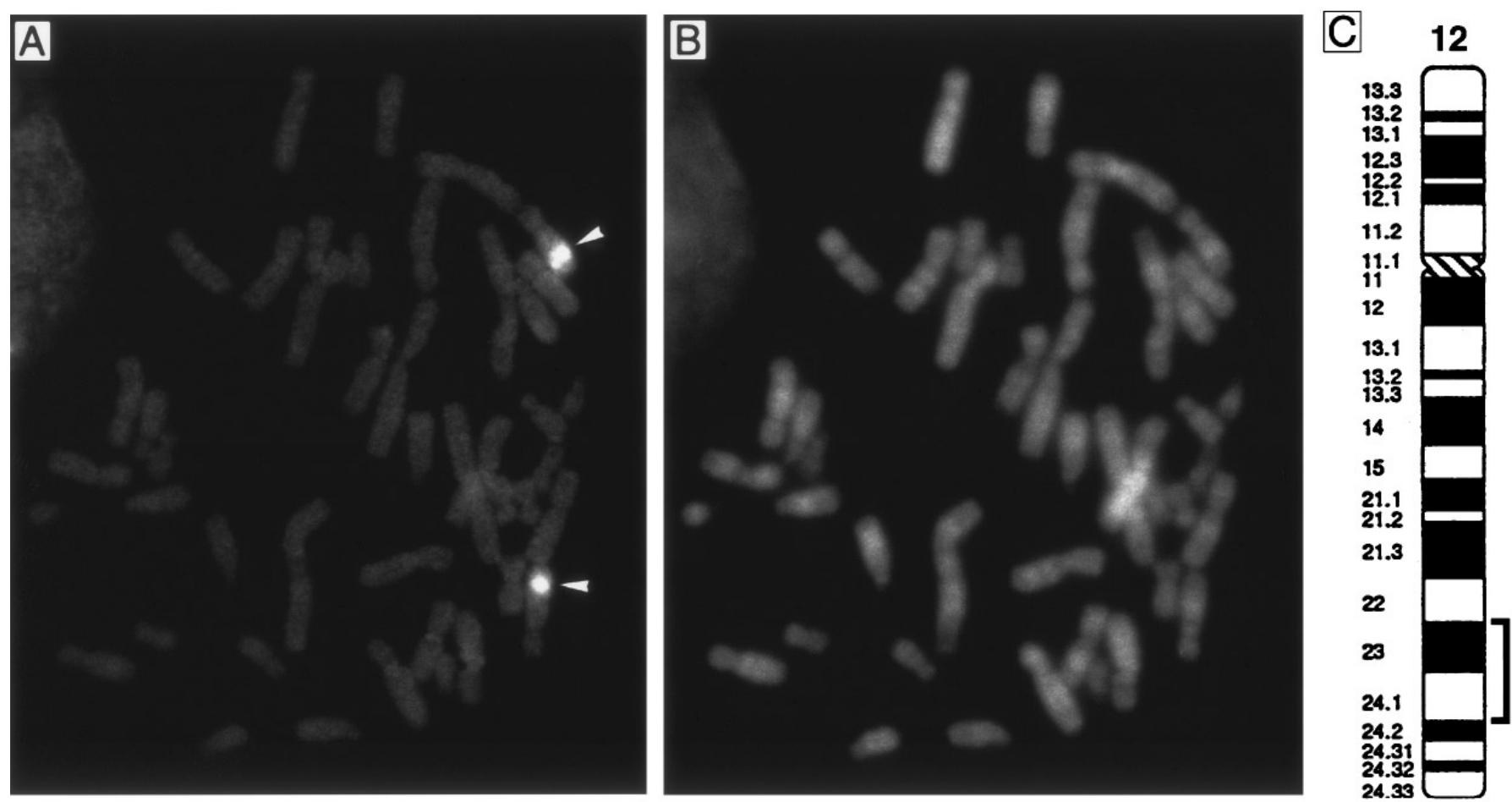

FIG. 4. In situ hybridization of metaphase chromosomes with biotinylated human photolyaselike cDNA probe (A) compared to DAPI banding of the same metaphase (B). The arrowheads indicate the hybridization signal localized on chromosome 12q23-q24.1. 
(Small et al., 1995). Interestingly, the amino acid sequence in the $\mathrm{C}$-terminal region of the human protein shows some homology with the C-terminus of the $\mathrm{C}$. reinhardtii photolyaselike protein (Fig. 1).

A phylogenetic tree (Fig. 2) was constructed based on amino acid sequences (without the $\mathrm{N}$ - or C-terminal extensions) of all known photolyases, confirming the assignment of the cloned human gene as a class I photolyase. Within this class, the human photolyase homolog forms a cluster together with the blue-light receptors, in accordance with the presence of a C-terminal extension.

\section{Tissue Expression}

Northern blot analysis of various human tissues was carried out using a radiolabeled PCR fragment from the cloned gene as probe (Fig. 3). Expression of mRNA of the gene is most prominent in testis followed by skeletal muscle, pancreas, and kidney, although constitutive expression was found in all tissues examined. Only a weak signal was found for leukocytes, although photoreactivating activity has been reported for these cells (Sutherland and Bennett, 1995). In additional experiments we found that the level of the gene expression in skin is as high as that in liver or brain (not shown) and less than that in brain or testis. The expression pattern suggests that the gene product may be involved in a function(s) other than repair of UV-damaged DNA. The other function(s) could be related to signal transduction like the plant blue-light photoreceptors. Photolyase activity of the gene product, however, remains to be determined. Two transcripts are observed (Fig. 3) and thought to be the results of alternative polyadenylation or splicing. The relative intensity of the two transcripts differs from tissue to tissue. In pancreas and liver the longer transcripts are relatively more abundant than in skeletal muscle and lung. The meaning of this differentiated expression is not known yet.

Southern analysis of genomic DNA from various organisms (Fig. 4) shows that homologs of the cloned human gene are present in a variety of organisms. This indicates the existence of a gene family related to photolyase-like blue-light receptors.

\section{Chromosomal Localization}

In situ hybridization was performed to determine the chromosomal localization of this gene. Chromosome 12q23-q24.1 was unequivocally identified as the site hybridizing to the cloned human gene (Fig. 5). In this region loci for Schinzel syndrome (ulnar-mammary syndrome, MIM No. 181450) and Darier-White disease (keratosis follicularis, MIM No. 124200) that may be related to the cloned gene have been mapped. No known human DNA repair-related syndromes or disorders seem to be associated with this locus as determined by an OMIM search (Pearson et al., 1994).

Very recently, during preparation of the manuscript, the sequence of a (6-4) photoproduct-specific photolyase from Drosophila melanogaster was published (Todo et al., 1996). A human homolog that is identical to the gene we cloned from homology to Anacystis photolyase was found in a database. It appears that the human sequence is even more similar to (6-4)photoproduct photolyase than to blue-light receptors.

\section{ACKNO WLEDGMENTS}

We are grateful to Dr. A. Hagemeijer and Miss E. van Drunen (Department of Cytogenetics, EUR) for chromosomal localization, to Miss I. Chiba for sequencing, and to Drs. J . H. J . Hoeijmakers and M. J . McKay for hel pful discussions and critical reading of the manuscript. This work was supported in part by a grant of the Research School of the Medical Genetics Centre South-West Netherlands, by the European Economical Community, Project F13PCT920007, and by a Grant-in-Aid for Scientific Research on Priority Areas from the J apanese Government to A.Y.

\section{REFERENCES}

Ahmad, M., and Cashmore, A. R. (1993). Hy4 gene of A.thaliana encodes a protein with characteristics of a blue-light photoreceptor. Nature 366: 162- 166.

Altschul, S. F., Gish, W., Miller, W., Myers, E. W., and Lipman, D. J . (1990). Basic local alignment search tool. J . Mol. Biol. 215: 403410.

Batschauer, A. (1993). A plant gene for photolyase: An enzyme catalyzing the repair of UV-light-induced DNA damage. Plant J . 4: 705- 709.

Chen, J -J ., Mitchell, D. L., and Britt, A. B. (1994). A light-dependent pathway for the elimination of UV-induced pyrimidine (6-4) pyrimidinone photoproducts in Arabidopsis. Plant Cell 6: 1311-1317.

Eker, A. P. M., Hessels, J . K. C., and van de Velde, J . (1988). Photoreactivating enzyme from the green alga Scenedesmus acutus. Evidence for the presence of two different flavin chromophores. Biochemistry 27: 1758-1765.

Felsenstein, J . (1993). "PHYLIP," Version 3.5c, Department of Genetics, University of Washington, Seattle, WA.

Kato, T., Todo, T., Ayaki, H., Ishizaki, K., Morita, T., Mitra, S., and I kenaga, M. (1994). Cloning of a marsupial DNA photolyase gene and the lack of related nucl eotide sequences in placental mammals. Nucleic Acids Res. 22: 4119-4124.

Kim, S-T., Li, Y. F., and Sancar, A. (1992). The third chromophore of DNA photolyase: Trp-277 of Escherichia coli DNA photolyase repairs thymine dimers by direct electron transfer. Proc. Natl. Acad. Sci. USA 89: 900-904.

Li, Y. F., Heelis, P. F., and Sancar, A. (1991). Active site of DNA photolyase: Tryptophan-306 is the intrinsic hydrogen atom donor essential for flavin radical photoreduction and DNA repair in vitro. Biochemistry 30: 6322-6329.

Li, Y. F., Kim, S. T., and Sancar, A. (1993). Evidence for lack of DNA photoreactivating enzyme in humans. Proc. Natl. Acad. Sci. USA 90: 4389-4393.

Malhotra, K., Kim, S-T. and Sancar, A. (1994). Characterization of a medium wavelength type DNA photolyase: Purification and properties of photolyase from Bacillus firmus. Biochemistry 33: 87128718.

O'Connor, K. A., MaBride, M. J ., West, M., Yu, H., Trinh, L., Yan, K., Lee, T., and Zusman, D. R. (1996). Photolyase of Myxococcus xanthus, a Gram-negative eubacterium, is more similar to photolyases found in archae and "higher" eukaryotes than to photolyases of other eubacteria. J . Biol. Chem. 271: 6252-6259.

Pearson, P., Francomano, C., Foster, P., Bocchini, C., Li, P., and McKusick, V. (1994). The status of online Mendelian inheritance in man (OMIM) medio 1994. Nucleic Acids Res. 22: 3470-3473. 
Pinkel, D., Straume, T., and Gray, J . W. (1986). Cytogenetic analysis using quantitative, high-sensitivity, fluorescence hybridization. Proc. Natl. Acad. Sci. USA 83: 2934- 2938.

Sancar, A. (1994). Structure and function of DNA photolyase. Biochemistry 33: 2-9.

Small, G. D., Min, B., and Lefebvre, P. A. (1995). Characterization of a Chlamydomonas reinhardtii gene encoding a protein of the DNA photolyase/blue light photoreceptor family. Plant Mol. Biol. 28: $443-454$.

Soares, M. B., de Fatima Bonaldo, M., J elene, P., Su, L., Lawton, L., and Efstratiadis, A. (1994). Construction and characterization of a normalized cDNA library. Proc. Natl. Acad. Sci. USA 91: 92289232.

Sutherland, B. M., and Bennett, P. V. (1995). Human white blood cells contain cyclobutyl pyrimidine dimer photolyase. Proc. Natl. Acad. Sci. USA 92: 9732-9736.
Todo, T., Takemori, H., Ryo, H., I hara, M., Matsunaga, T., Nikaido, O., Sato, K., and Nomura, T. (1993). A new photoreactivating enzyme that specifically repairs ultraviolet light-induced (6-4) photoproducts. Nature 361: 371- 374.

Todo, T., Ryo, H., Yamamoto, K., Toh, H., Inui, T., Ayaki, H., Nomura, T., and Ikenaga, M. (1996). Similarity among the Drosophila (6-4)photolyase, a human photolyase homolog, and the DNA photolyase-blue-light photoreceptor family. Science 272: 109- 112.

Yasui, A., Yajima, H., Kobayashi, T., Eker, A. P. M., and Oikawa, A. (1992). Mitochondrial DNA repair by photolyase. Mutat. Res. 273: $231-236$.

Yasui, A., Eker, A. P. M., Yasuhira, S., Yajima, H., Kobayashi, T., Takao, M. and Oikawa, A. (1994). A new class of DNA photolyases present in various organisms including aplacental mammals. EMBO J . 13: 6143-6151. 